Manu Bala Krishnan, Samuel Rosset, Shanti Bhattacharya, and Herbert R. Shea

\title{
Fabrication of transmissive dielectric elastomer actuator driven tunable optical gratings with improved tunability
}

Opt. Eng. 55(4), 047104 (Apr 12, 2016).

http://dx.doi.org/10.1117/1.OE.55.4.047104

Copyright 2016 Society of Photo Optical Instrumentation Engineers. One print or electronic copy may be made for personal use only. Systematic reproduction and distribution, duplication of any material in this paper for a fee or for commercial purposes, or modification of the content of the paper are prohibited. 


\section{Optical Engineering}

\section{Fabrication of transmissive dielectric elastomer actuator driven tunable optical gratings with improved tunability}

Manu Bala Krishnan

Samuel Rosset

Shanti Bhattacharya

Herbert R. Shea 


\title{
Fabrication of transmissive dielectric elastomer actuator driven tunable optical gratings with improved tunability
}

\author{
Manu Bala Krishnan, ${ }^{a, *}$ Samuel Rosset, ${ }^{b}$ Shanti Bhattacharya, ${ }^{a}$ and Herbert R. Shea ${ }^{b}$ \\ andian Institute of Technology Madras, Department of Electrical Engineering, Chennai, Tamil Nadu 600036, India \\ ${ }^{b}$ Ecole Polytechnique Fédérale de Lausanne, Microsystems for Space Technologies Laboratory, 2002 Neuchâtel, Switzerland
}

\begin{abstract}
We present an electrically actuated tunable optical grating based on dielectric elastomer actuators. A simple fabrication protocol is presented, which integrates the grating with the actuating mechanism both made of soft elastomers, improving the tunability of the grating. The device is designed to be operated in the transmission mode. It exhibits a continuous period tunability of $34.4 \%$ at an actuation voltage of $5.5 \mathrm{kV}$, which is an improvement over reported tunable optical gratings. @ 2016 Society of Photo-Optical Instrumentation Engineers (SPIE) [DOI: 10.1117/1.OE.55.4 .047104]
\end{abstract}

Keywords: dielectric elastomer actuators; diffraction; tunable optics.

Paper 151784 received Dec. 17, 2015; accepted for publication Mar. 18, 2016; published online Apr. 12, 2016.

\section{Introduction}

Tunable optical gratings are used for a wide range of applications, including spectroscopy, ${ }^{1}$ displays, ${ }^{2}$ and optical communication systems. ${ }^{3}$ The ability to tune the period over a large range is an important feature for all these optical applications. Silicon-based microfabrication technology is widely used for tunable grating devices. ${ }^{4-6}$ However, such devices have limited tuning range. $12.5 \%$ tuning has been reported for an electrostatically actuated micro electro mechanical systems (MEMS) device. ${ }^{7}$ Thermally actuated MEMS devices ${ }^{8}$ can offer improved tuning up to $25 \%$ but are limited by slow response time and high power consumption. Another approach for highly tunable optical devices is fluidic actuation of elastomer membranes. ${ }^{9}$ Elastomer grating devices with fluidic actuation with $30 \%$ period tunability have been reported. ${ }^{10}$ Performance is limited by beam profile distortion due to out-of-plane actuation. Also, actuation speed is constrained by the fluid injection mechanism. A promising actuation alternative is provided by dielectric elastomer actuators (DEAs), due to their ability to generate large strains at high speeds, the speed of the response being limited typically by viscoelastic losses and electrical response time. ${ }^{11}$ They have been employed in diverse areas such as micropumps, ${ }^{12}$ robotics, ${ }^{13}$ and haptic devices. In addition to enabling large strains, the optical transparency of the elastomers in the visible range has made it possible to realize phase retarders, ${ }^{14}$ tunable lenses, ${ }^{15,16}$ and tunable gratings. ${ }^{17-23}$

We present here a silicon-based transmission grating with an improved period tunability of $34.4 \%$, which we achieved with an improved design and fabrication technique. The majority of the DEA-based devices reported in this field consist of acrylic-based actuators onto which a separately fabricated soft grating is bonded. In contrast, our device contains the grating as an integral part of the actuator, with all parts being made of silicon elastomer. DEAs based on the 3M acrylic very high bond (VHB) have demonstrated snap-through area strain up to $1692 \%^{24}$ and

*Address all correspondence to: Manu Bala Krishnan, E-mail: manubalakrishnan@gmail.com generally offer several times higher strain than siliconbased devices. However, VHB suffers from high viscoelastic losses and creep compared with silicon elastomers, and its lifetime is limited due to the high prestretch required. Although not capable of delivering as high a strain as VHB, silicon elastomers exhibit much faster electromechanical response and much better position stability. ${ }^{25}$ Fast and accurate tuning of optical parameters, such as focal length and grating period, are essential for tunable optical devices. Also important is the ability to hold to a stable state for an extended time. These requirements are better met by silicon elastomers, ${ }^{16}$ which have been our choice as membrane material for the present work. The generally lower actuation strain obtained with silicon membranes is addressed by our design and fabrication protocol, which allow our device to exhibit strains comparable with what has been achieved with VHB-based tunable gratings. Designed to be operated in the transmission mode, the device contains no reflective layers, which would stiffen the actuator. This, combined with the reduced stiffness obtained thanks to the integration of the grating into the actuator membrane, allows reaching larger tuning ranges. Therefore, even though the actuator is based on a silicon membrane, our device can exhibit up to $34.4 \%$ of tuning range, which is much higher than most published results on $\mathrm{VHB},{ }^{18,20}$ and can perform better than the highest tuning range of $32 \%$ reported on VHB. ${ }^{22}$

\subsection{Principle of Operation}

Our device consists of a prestretched elastomer membrane with sinusoidal surface profile on the top surface and two sets of printed electrodes, leaving the central region of the membrane to be used as an optical grating (see Fig. 2). The electrode regions expand when electrostatically actuated (illustrated in Fig. 1), and this produces a compressive strain on the grating at the center, thereby changing the grating pitch. The operation of a diffraction grating in normal incidence is described by the grating equation $d \sin \theta_{m}=m \lambda$, where $\theta_{m}$ denotes the diffraction angle of the $m$ 'th order 


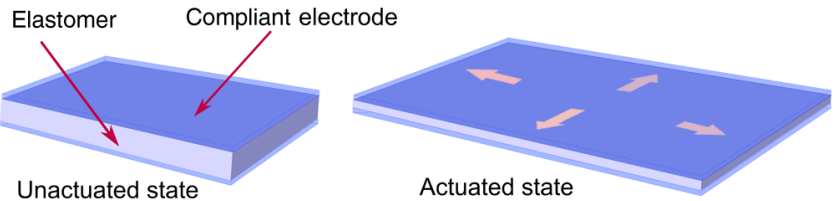

Fig. 1 Working principle of a DEA.

for a grating with period $d$ and with light of wavelength $\lambda$ incident on it. A detector positioned at a given $\theta$ will detect different wavelengths present in the incident beam as the period is tuned.

The working principle of a DEA is depicted in Fig. 1. An elastomer layer is sandwiched between a pair of compliant electrodes. When a voltage $V$ is applied across the electrodes, due to Maxwell's stress, ${ }^{26-28}$ a thickness strain $S_{z}=-\left(\varepsilon V^{2} / Y t^{2}\right)$ is experienced by the sandwiched membrane. Here, $t$ is the thickness of the membrane, $\varepsilon$ is the electric permittivity, and $Y$ is the Young's modulus of the material. This equation for thickness strain is valid only for small strains, and the thickness can be assumed to be the initial thickness of the membrane. Assuming that the membrane is incompressible, the strains in planar directions are related to $S_{z}$ through the following equation:

$\left(1+S_{x}\right)\left(1+S_{y}\right)\left(1+S_{z}\right)=1$.

If the strains in planar dimensions are taken to be the same, i.e., $S=S_{x}=S_{y}$, the equation reduces to

$S=\left(\sqrt{1-\left(\varepsilon V^{2} / Y t^{2}\right)}\right)^{-1}-1$.

As the membrane becomes thinner due to the thickness strain, its area increases. If an optical grating is present on top of the membrane, the grating period increases with the applied voltage. With a reflective layer coated on the grating, this configuration has been employed for fabrication of reflective tunable gratings. ${ }^{22}$ To fabricate a transmission grating, a scheme such as the one shown in Fig. 2 is used in our device. In this configuration, two pairs of electrodes define the active region of the device. The membrane is now constrained on the boundaries with the help of frames, so that the induced strain is concentrated in the passive region at the center. Furthermore, the membrane is stretched prior to attaching the fixed frame. Once the active regions expand on actuation, compressive strain is developed in the passive region, which could buckle the membrane and deform the

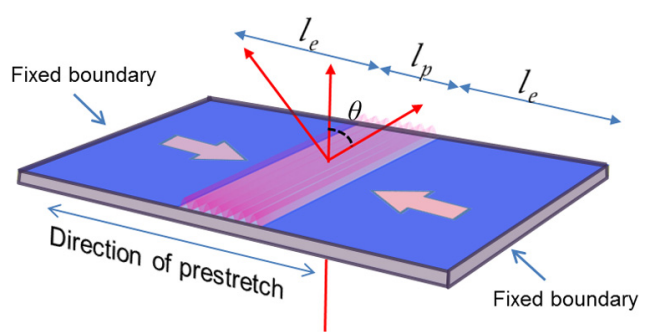

Fig. 2 Actuation of the grating by the two-electrode configuration. As voltage increases, the period reduces and the first-order diffraction angle increases. The grating lines are aligned normal to the direction of actuation. gratings. Prestretching the membrane ensures that the membrane is already in tensile stress, thereby avoiding buckling during actuation. Also, the prestretch eliminates the electromechanical instability of the membrane and allows for high actuation strain. ${ }^{29}$ Upon actuation, the electrodes expand and the passive region contracts in length, thereby reducing the period of grating in this region. The strain in the passive region is considered the negative of $S$, modified by a geometric parameter that depends on the electrode geometry. In Fig. 2 , the total length $\left(2 l_{e}+l_{p}\right)$ remains constant due to the fixed boundary condition. Due to a strain $S$, the elongation of each electrode is $S l_{e}$, and to maintain the total length constant, the passive region compresses to a length $l_{p}\left[1-2 S\left(l_{e} / l_{p}\right)\right]$. In terms of the grating period, the period $d$ at a strain $S$ can be expressed as

$d=d_{0}(1-k S)$

where $d_{0}$ is the period of the grating in the unactuated state and $k$ is the geometric parameter given by

$k=2 l_{e} / l_{p}$.

The tunable grating device discussed here works in the transmission mode with the passive central region illuminated at normal incidence.

The configuration shown in Fig. 2 has been used in the design of other tunable transmissive gratings, but with the grating added as a separate layer bonded to an actuator membrane. ${ }^{18,21,23}$ The drawback of this approach is that the grating stiffens the actuator and therefore the maximum induced strain of the actuator is reduced compared with the bare actuator. In this work, we developed a process where the grating layer is intrinsically part of the actuator membrane, thereby enhancing the tunability of the grating by eliminating the stiffening impact of the grating.

\section{Fabrication of the Device}

\subsection{Fabrication}

The tunable grating device is made from two membranes separately cast on flexible temporary substrates (Fig. 3). One of the membranes is cast on a master-grating sheet, seen in Fig. 3(a), so that the grating profile is replicated on the membrane once cured. Holographic grating sheets from Rainbow Symphony with sinusoidal grating profiles were used as the master grating. The master grating is silanized prior to the casting of membrane. This inhibits the adhesion between the silicon membrane and the grating sheets, thereby making the release of the cured membrane easier. The second membrane is cast on a substrate of polyethylene terephthalate (PET) foil with an intermediate layer of poly (acrylic acid) (PAA) used as sacrificial layer, allowing the separation of the silicon membrane from the PET substrate. Both membranes are made by mixing parts $\mathrm{A}$ and $\mathrm{B}$ of the silicon Nusil CF 18-2186 in the ratio 1:1 with an equal amount by weight of a siloxane-based solvent and are cured at $80^{\circ} \mathrm{C}$ for $2 \mathrm{~h}$ after casting. The second membrane is cut into circular pieces and is transferred to a temporary flexible frame. The PET substrate is removed from the membrane by immersing in hot DI water, which dissolves the intermediate PAA layer. The grating membrane is then oxygen-plasma bonded to this membrane with a drop of 

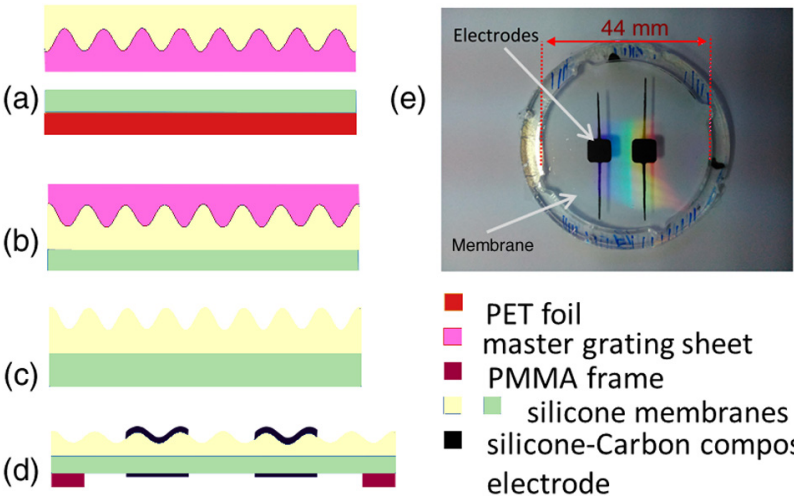

PET foil

master grating sheet

- PMMA frame

silicone membranes

- silicone-Carbon composite electrode

Fig. 3 Fabrication process flow of the device: (a) silicon membranes separately cast on master grating and PET foil; (b) grating is bonded to the free-released silicon membrane; (c) master grating is peeled off; (d) the membrane is stretched and fixed to a rigid PMMA frame, and carbon black electrodes are printed; and (e) final device showing the membrane dimension of $44 \mathrm{~mm}$ in diameter.

isopropyl alcohol between the membranes to avoid curling and formation of air traps while bonding. Once the membranes are well bonded, as shown in Fig. 3(b), the master grating is peeled off, leaving behind the structure shown in Fig. 3(c). The method of bonding two membranes to form the devices helps in effortless removal of the master grating from the membrane. The grating membrane is firmly supported by the bonded membrane on one side, holding the grating membrane in position, thus avoiding curling and damage to the grating membrane while the master grating is removed from the other side. The membrane is equibiaxially prestretched and attached to a rigid PMMA frame [Fig. 3(d)]. Electrodes are contact printed on the top and bottom sides of the membrane in alignment with the grating lines (Fig. 2). The electrode material is prepared by mixing carbon black in silicon, as detailed in Ref. 30, and is cured in an oven at $80^{\circ} \mathrm{C}$ for $30 \mathrm{~min}$ after the contact printing.

The surface profile of the original and replicated gratings (on the membrane) is shown in Fig. 4. The profiles were not measured at the same location of the master grating and the membrane. The average grating height of the master grating is $0.219 \mu \mathrm{m}$ with a standard deviation of $0.017 \mu \mathrm{m}$. The numbers are 0.198 and $0.016 \mu \mathrm{m}$, respectively, for the replicated grating, clearly indicating the success of the replication process. After the device is fabricated, the membrane is $38 \mu \mathrm{m}$ thick.

\subsection{Design Optimization}

The performance of the device is largely determined by the prestretch of the silicon membrane, the geometry of the electrode, and its position on the membrane. ${ }^{31}$ The effect of both the prestretch and the geometry was studied experimentally, and the optimum values, which provide the maximum compressive strain in the passive region, were used to fabricate the final set of devices. These optimization steps were carried out on single-membrane devices that did not contain gratings. The grating depth being small compared with the total thickness of the device (less than 1\%), the final devices with gratings show the same behavior on actuation. The devices were actuated with voltage values, ramped up in steps from zero, up to their breakdown voltages, at which point the devices fail. The induced compressive strain in the passive region was calculated by observing the change
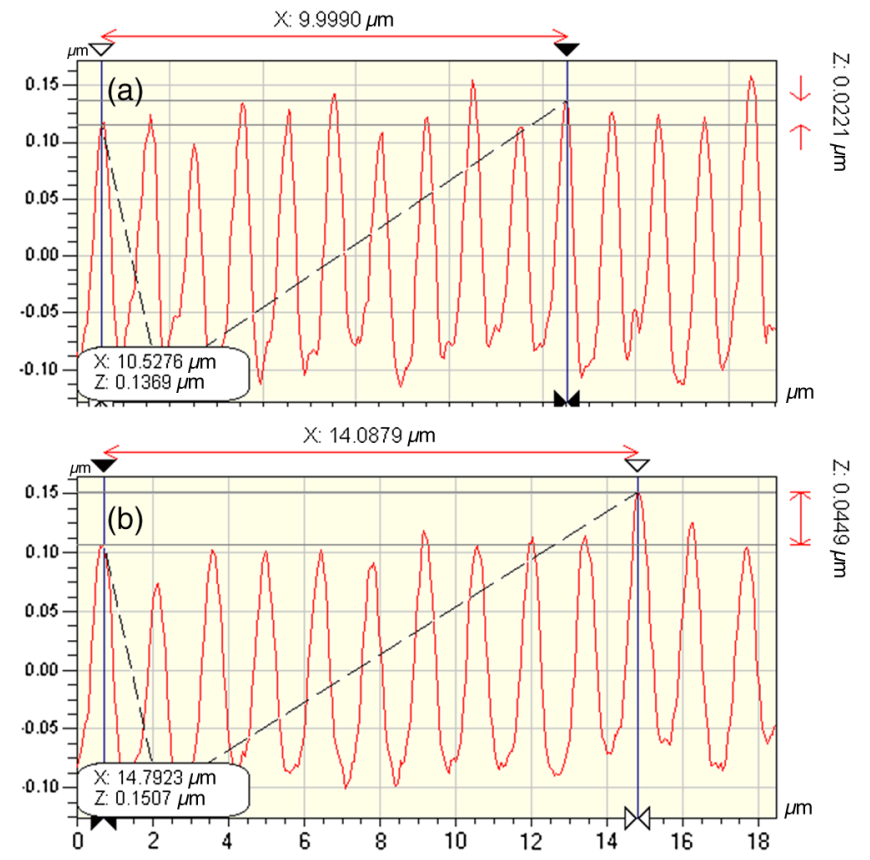

Fig. 4 Optical profile of the cross-section of (a) master grating and (b) prestretched membrane.

in position of the edges of the electrodes during actuation as captured on a camera. To study the effect of prestretch, devices with prestretch ratio varying from 1.2 to 1.9 of the original dimension were fabricated, keeping the shape and size of electrodes the same on all the devices. For devices with prestretch ratio up to 1.4 , the maximum induced strain was observed to be not greater than $10 \%$. The induced strain improved up to $21.5 \%$ as the prestretch ratio was increased to 1.9. The response of the devices to an applied voltage follows the same trend for prestretch ratio of 1.5 and above (Fig. 5).

In another set of experiments, the effect of size and positioning of electrodes were studied. The area of the electrodes defines the passive region at the center available for illumination. As described by Eqs. (3) and (4), for any given prestretch of the membrane, the geometric parameter determines

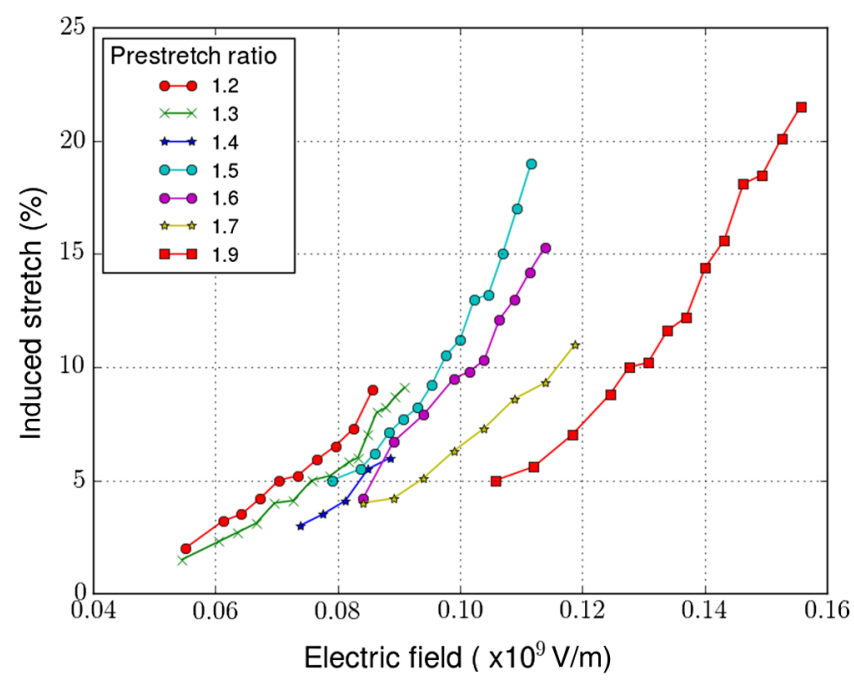

Fig. 5 Dependence of the electric field-stretch relation on the prestretch of the membrane. 

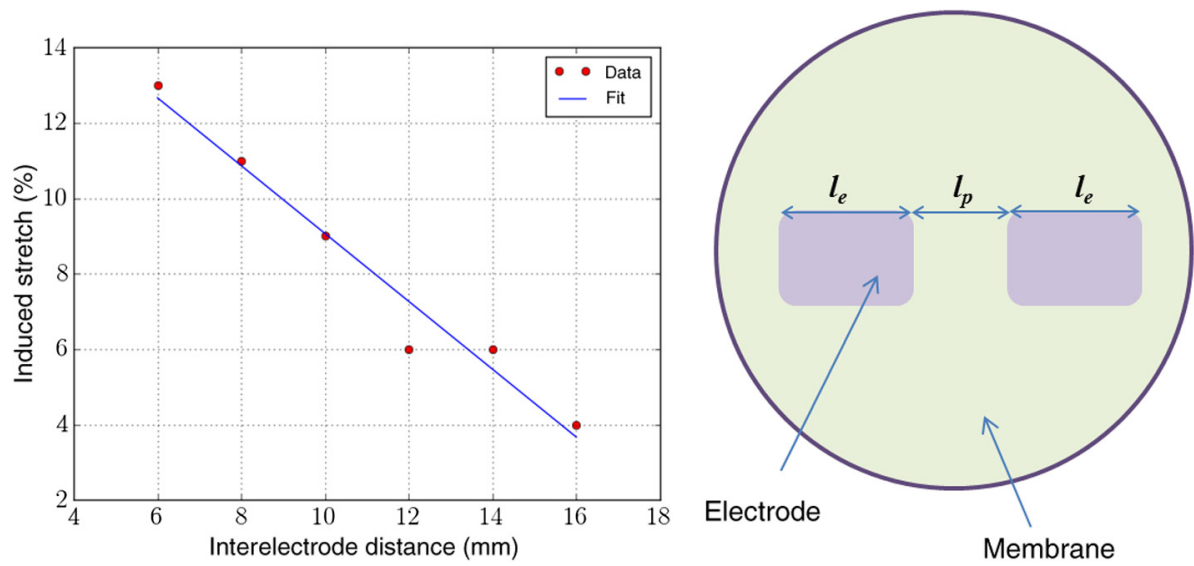

Fig. 6 The induced stretch in the central region of the membrane decreases as the interelectrode distance increases.

the compressive strain induced in passive region and therefore the tunability of the grating. In the experiment, $l_{p}$ is increased while keeping the total length $\left(2 l_{e}+l_{p}\right)$ a constant. As the separation $l_{p}$ is increased, the induced compressive strain in the passive region is found to decrease. For example, in a membrane with prestretch of 1.2 , the induced strain is $13 \%$ for the interelectrode distance of $6 \mathrm{~mm}$ and reduces to $4 \%$ when the electrodes are placed at a separation of $16 \mathrm{~mm}$ (Fig. 6).

The first-order diffraction efficiency of a sinusoidal phase grating is expressed in terms of the first-order Bessel function $\left[\left[J_{1}(m / 2)\right]^{2}\right.$ for an optical phase delay of $m=2 \pi(n-1) s / \lambda$, where $n$ is the refractive index $(1.445$ for Nusil CF 18-2186), $s$ is the grating height, and $\lambda$ is the wavelength. To obtain the maximum possible efficiency of $33.8 \%$, a grating height of $0.833 \mu \mathrm{m}$ is required at a wavelength of $632.8 \mathrm{~nm}$. The grating master used in the fabrication had a height of $0.219 \mu \mathrm{m}$, which means a diffraction efficiency of $5 \%$. Even though the grating master chosen for the fabrication is not ideal in terms of diffraction efficiency, it is readily available and serves the purpose of demonstrating the fabrication process.

\section{Results and Discussion}

\subsection{Optical Characterization Results}

The devices were optically tested for the tunability of the grating period. The central region of the device is illuminated with a He-Ne laser $(632.8 \mathrm{~nm})$ at normal incidence. The transmitted diffraction pattern is allowed to fall on a screen normal to the input laser beam. The DC voltage, applied across the pair of electrodes, is swept from $0 \mathrm{~V}$ up to the breakdown voltage of the device in discrete steps. The period of the grating for each applied voltage is calculated from the observed position of the first-order diffraction spot on the screen, with respect to the zeroth order. Devices made from master gratings of period $1 \mu \mathrm{m}$ were tested. The grating period varies from the prestretched value of $1.42 \mu \mathrm{m}$ at $0 \mathrm{kV}$ to a final value of $1.06 \mu \mathrm{m}$ at $5.5 \mathrm{kV}$ (Fig. 7). This corresponds to a tuning range of $34.4 \%$, with the grating period of the nonprestretched membrane as a reference. The observed tuning of the device follows the theoretical curve [Eq. (3)], with a geometric parameter of $k=0.65$ (Fig. 7).
A common drawback with elastomer-based tunable grating is that with the tuning of the grating period, the grating height is also modulated during actuation. This changes the diffraction efficiency of the grating with applied voltage. As it was not experimentally possible to measure the surface profile of the grating during actuation, an estimate of the change in first-order diffraction efficiency was carried out as follows. Surface profiling of the device, in its unactuated state (grating period of $1.42 \mu \mathrm{m}$ ), shows a grating height of $0.198 \mu \mathrm{m}$, while the original master grating has a height of $0.219 \mu \mathrm{m}$. Therefore, the maximum possible change in grating height during actuation would be $21 \mathrm{~nm}$, which means a change in first-order efficiency $\left[J_{1}(\mathrm{~m} / 2)\right]^{2}$ of only $0.95 \%$. The observed first-order efficiency did not vary much during the entire range of the tuning of the grating, and the expected change was not captured by the photodetector used.

The profiles of the first-order diffracted beams for a device with prestretch ratio of 1.2 were captured with a CCD camera. The beam retains its original profile up to $5 \mathrm{kV}$. Beyond this value, it starts distorting, which indicates a deformation of the grating at voltages higher than $5 \mathrm{kV}$ (Fig. 8). The deformation of the grating profile can be attributed to increased nonlinearity in mechanical behavior of the

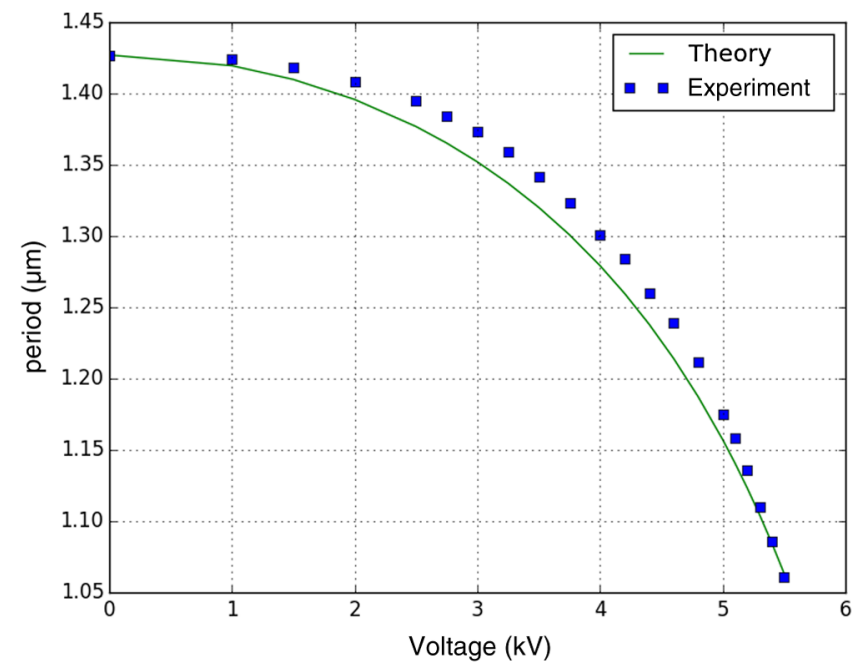

Fig. 7 Tunability of the grating period for different voltages. 


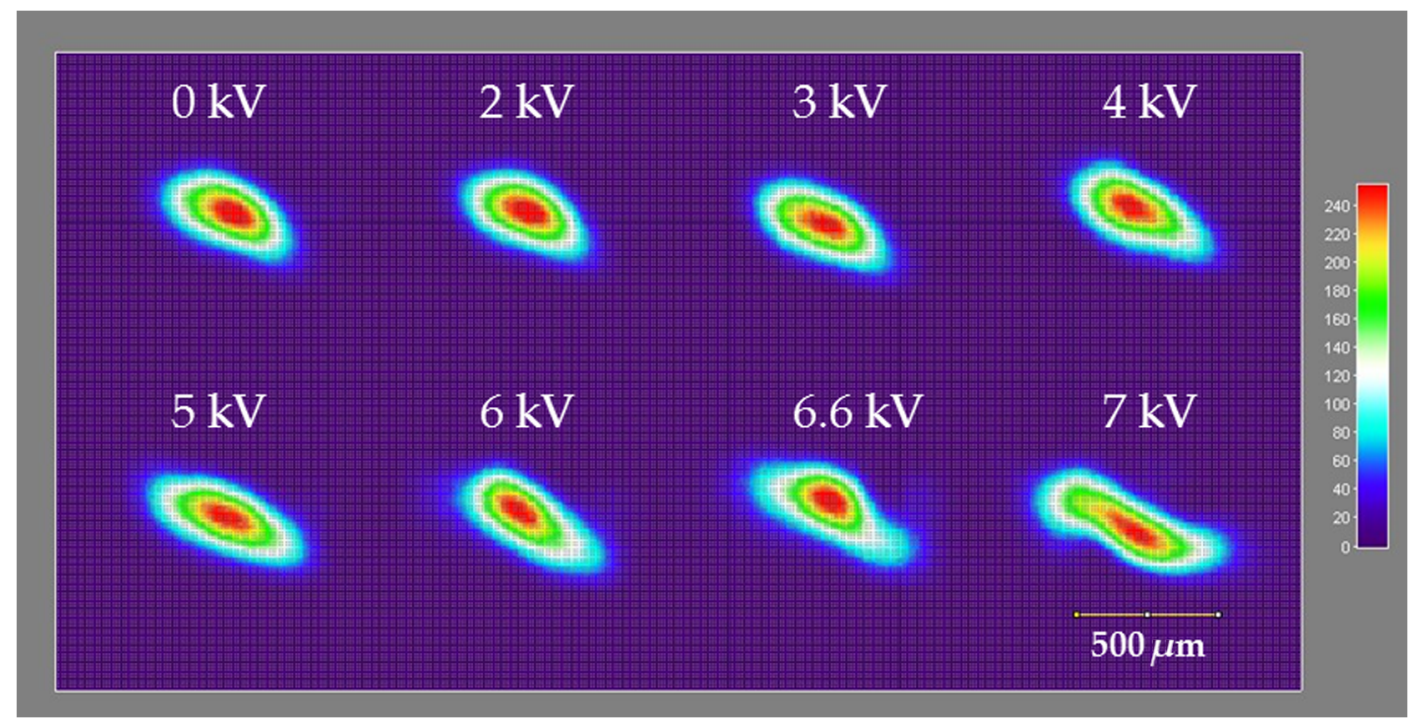

Fig. 8 Profile of the first-order diffracted beam at various actuation voltages for a device with prestretch ratio 1.2 .

material at high voltages. The mechanism of beam profile deformation needs to be explored further.

\section{Conclusions}

We have fabricated and characterized a transmission optical grating for the visible range whose period can be tuned based on the DEAs. A simple and efficient fabrication technique has been developed. The grating period of the device demonstrates an analog tuning of $34.4 \%$ at a voltage of $5.5 \mathrm{kV}$. This tuning range is the highest reported in the class of tunable optical gratings driven by DEA. The higher tuning range is obtained as a result of the way the grating is fabricated. Unlike previously reported approaches, where the grating is bonded onto a prestretched acrylic actuator membrane, our device has the advantage that the grating is an integral part of the actuated membrane, simplifying processing, but more importantly adding very little stiffness to the device. As the device is designed for use in transmission mode, no reflective coatings are required on the membrane, which would increase the membrane stiffness. Our method has made it possible to fabricate highly tunable gratings with silicon elastomers, exploiting their optical quality, low viscoelasticity, and rapid response, despite their lower actuation strain compared with acrylic elastomers used in earlier approaches. ${ }^{18-20,22}$ The height of the grating, which determines the diffraction efficiency, varies very little during actuation.

In this work, off-the-shelf master gratings were used in the fabrication process, which resulted in poor efficiency due to their unoptimized height. The first-order efficiency can be increased substantially by having master gratings fabricated with appropriate values for the initial grating height. Devices with high optical efficiency can be made by employing master gratings of suitable dimensions and grating profiles. Since silanization works well with oxidized silicon surfaces, silicon substrates with microfabricated grating profiles are a good choice for high-efficiency tunable devices. The fabrication protocol can easily be extended to elastomer materials of higher dielectric constant and membranes of lower thickness, thereby improving the tunability and bringing down the actuation voltage. The dimensions that offer the best tuning of the device were fixed by independently optimizing the value of prestretch of the polymer membrane and electrode dimensions. There is a scope of further increasing the tunability of the device by varying the parameters of electrode geometry and the nature of prestretch.

\section{Acknowledgments}

MK thanks the EPFL for funding his visit to the EPFLLMTS. He also thanks the members of the EPFL-LMTS who helped with device fabrication. MK and SB thank the CNNP, IIT Madras, for the use of their characterization facility.

\section{References}

1. S. C. Truxal, K. Kurabayashi, and Y.-C. Tung, "Design of a MEMS tunable polymer grating for single detector spectroscopy," Int. J. Optomechatronics 2(2), 75-87 (2008).

2. D. M. Bloom, "Grating light valve: revolutionizing display technology," Proc. SPIE 3013, 165-171 (1997).

3. G. Huang et al., "Surface relief apodized grating tunable filters produced by using a shadow mask," Opt. Express 23, 21090-21096 (2015).

4. M. Tormen et al., "Deformable MEMS grating for wide tunability and high operating speed," J. Opt. A: Pure Appl. Opt. 8(7), S337-S340 (2006).

5. D.-Y. Qiao et al., "A resonance scanning grating based on SOI for microspectrometer application," Laser Phys. 23(3), 035601 (2013).

6. C. W. Wong et al., "Analog tunable gratings driven by thin-film piezoelectric microelectromechanical actuators," Appl. Opt. 42(4), 621-626 (2003).

7. L. Xiang et al., "A compressed wide period-tunable grating working at low voltage," J. Semicond. 31, 104010 (2010).

8. Y. S. Yang et al., "A large-displacement thermal actuator designed for MEMS pitch-tunable grating," J. Micromech. Microeng. 19(1), 015001 (2008)

9. G. Zhou et al., "Liquid tunable diffractive/refractive hybrid lens," $O p t$ Lett. 34, 2793-2795 (2009)

10. R. A. Guerrero, S. J. C. Oliva, and J. M. M. Indias, "Fluidic actuation of an elastomeric grating," Appl. Opt. 51, 5812-5817 (2012).

11. R. Pelrine, "High-speed electrically actuated elastomers with strain greater than 100\%," Science 287(5454), 836-839 (2000).

12. J. J. Loverich, I. Kanno, and H. Kotera, "Concepts for a new class of all-polymer micropumps," Lab Chip 6(9), 1147-1154 (2006).

13. G. Kovacs, P. Lochmatter, and M. Wissler, "An arm wrestling robot driven by dielectric elastomer actuators," Smart Mater. Struct. 16(2), s306-s317 (2007)

14. M. Beck, R. Fiolka, and A. Stemmer, "Variable phase retarder made of a dielectric elastomer actuator," Opt. Lett. 34(6), 803-805 (2009). 
15. S. Shian, R. M. Diebold, and D. R. Clarke, "Tunable lenses using transparent dielectric elastomer actuators," Opt. Express 21(7), 8669-8676 (2013)

16. L. Maffli et al., "Ultrafast all-polymer electrically tunable silicone lenses," Adv. Funct. Mater. 25(11), 1656-1665 (2015).

17. S. Rosset et al., "Tunable grating with active feedback," Proc. SPIE 8687, 86872F (2013).

18. M. Kollosche et al., "Voltage-controlled compression for period tuning of optical surface relief gratings," Opt. Lett. 36(8), 1389-1391 (2011).

19. M. Aschwanden and B. A. Stemmer, "Diffractive transmission grating tuned by dielectric elastomer actuator," IEEE Photonics Technol. Lett. 19(14), 1090-1092 (2007).

20. M. Aschwanden and A. Stemmer, "Low voltage, highly tunable diffraction grating based on dielectric elastomer actuators," Proc. SPIE 6524, 65241N (2007).

21. S. Rosset et al., "Self-sensing dielectric elastomer actuators in closedloop operation," Smart Mater. Struct. 22(10), 104018 (2013).

22. M. Aschwanden and A. Stemmer, "Polymeric, electrically tunable diffraction grating based on artificial muscles," Opt. Lett. 31(17), 2610-2612 (2006)

23. M. Aschwanden, D. Niederer, and A. Stemmer, "Tunable transmission grating based on dielectric elastomer actuators," Proc. SPIE 6927, 69271R (2008).

24. C. Keplinger et al., "Harnessing snap-through instability in soft dielectrics to achieve giant voltage-triggered deformation," Soft Matter $\mathbf{8}(2)$ 285-288 (2012).

25. S. Michel et al., "A comparison between silicone and acrylic elastomers as dielectric materials in electroactive polymer actuators," Polym. Int. 59(3), 391-399 (2009).

26. S. J. A. Koh et al., "Mechanisms of large actuation strain in dielectric elastomers," J. Polym. Sci., Part B: Polym. Phys. 49(7), 504-515 (2011).

27. R. E. Pelrine, R. D. Kornbluh, and J. P. Joseph, "Electrostriction of polymer dielectrics with compliant electrodes as a means of actuation," Sens. Actuators, A 64(1), 77-85 (1998).

28. Z. Suo, "Theory of dielectric elastomers," Acta Mech. Solida Sin. 23(6), 549-578 (2010).

29. B. Li et al., "Effect of mechanical pre-stretch on the stabilization of dielectric elastomer actuation," J. Phys. D 44(15), 155301 (2011).

30. S. Rosset et al., "Fabrication process of silicone-based dielectric elastomer actuators," J. Visualized Exp. 108, e53423 (2016).
31. S. Rosset, O. A. Araromi, and H. R. Shea, "Maximizing the displacement of compact planar dielectric elastomer actuators," Extreme Mech. Lett. 3, 72-81 (2015).

Manu Bala Krishnan received his MSc degree in physics and MTech degree in optoelectronics and laser technology from Cochin University of Science and Technology, India, in 2004 and 2009, respectively. After working as a research assistant at the Indian Institute of Technology Bombay in the field of MEMS biosensors, he is at present pursuing his $\mathrm{PhD}$ in the Department of Electrical Engineering, IIT Madras, on MEMS tunable diffractive optics.

Samuel Rosset received his MS and PhD degrees in microengineering from the EPFL, Lausanne, Switzerland, in 2004 and 2009, respectively. After a few years in industry developing tunable optics based on soft elastomers, he reintegrated at EPFL in 2011, where he is now a research scientist working on dielectric elastomer actuators. His research interests include soft actuators, compliant electrodes, development of new fabrication processes for soft sensors and actuators, and reliability and lifetime of these devices.

Shanti Bhattacharya received her $\mathrm{PhD}$ in physics from the Indian Institute of Technology Madras in 1997 in the area of optical array illuminators. She then spent 3 years at the Technical University of Darmstadt, Germany, as a postdoctoral fellow. She later joined the MEMS division of Analog Devices, Cambridge, USA, where she worked on the design of an optical MEMS switch. She is currently an associate professor at IIT Madras.

Herbert R. Shea is an associate professor at the EPFL in Switzerland, leading a group developing elastomer-based actuators and sensors for applications including haptic displays, soft robotics, and optics and tools for mechanobiology. After his $\mathrm{PhD}$ in 1997 from Harvard University, he spent 2 years as a postdoc at IBM Research, then joined Lucent Technologies' Bell Labs, becoming the technical manager of the microsystems technology group. In 2004, he joined the EPFL as a faculty member. 